\title{
The PI3K/Akt/mTOR pathway is involved in CVB3-induced autophagy of HeLa cells
}

\author{
HUAN CHANG $^{1}$, XIN LI $^{1}$, QIAN CAI ${ }^{1}$, CHUNYUN LI $^{1}$, LANG TIAN $^{1}$, JIA CHEN $^{1}$, \\ XIAOWEI XING ${ }^{2}$, YU GAN ${ }^{3}$, WEN OUYANG ${ }^{4}$ and ZUOCHENG YANG ${ }^{1}$ \\ ${ }^{1}$ Department of Pediatrics, ${ }^{2}$ Center for Medical Experiments, Departments of ${ }^{3}$ Urology and ${ }^{4}$ Anesthesiology, \\ The Third Xiangya Hospital, Central South University, Changsha, Hunan 410013, P.R. China
}

Received June 3, 2016; Accepted May 26, 2017

DOI: $10.3892 /$ ijmm.2017.3008

\begin{abstract}
Recent studies have found that viral myocarditis (VMC) associated with coxsackievirus B3 (CVB3) causes autophagy activation after infection, but the specific mechanism is not clear. The present study demonstrated that the phosphatidylinositol 3-kinase (PI3K)/protein kinase $\mathrm{B}(\mathrm{PKB}) / \mathrm{mammalian}$ target of rapamycin (mTOR) signaling pathway participates in CVB3-induced autophagy. We found that the light chain 3 (LC3)-II/LC3-I ratio was increased and $\mathrm{p} 62$ and $\mathrm{p}-\mathrm{mTOR}$ were altered at different times during CVB3 infection. To further assess the effects of this signaling pathway on CVB3 infection and viral replication, we selected $24 \mathrm{~h}$ post-inoculation (h.p.i.) as our research time point to conduct our next study. We inhibited the function of PI3K, Akt1 and mTOR. The outcome showed that inhibition of PI3K with ZSTK474 alleviated autophagy and decreased CVB3 mRNA replication and VP1 expression. Inhibition of mTOR with rapamycin promoted autophagy and viral mRNA replication but did not impact VP1 expression. Inhibition of Akt with MK2206 aggravated autophagy induced by viral infection. In our research, p62 exhibited a decrease at the beginning of infection but then increased as infection time increased. This finding may serve as a clue to elucidate the function of autophagy at different times of infection. However, the details merit further study. In conclusion, our findings suggest that the PI3K/Akt/mTOR signaling pathway participates in the process of autophagy induced by CVB3 infection. This finding may provide a new perspective of CVB3-induced autophagy.
\end{abstract}

Correspondence to: Professor Zuocheng Yang, Department of Pediatrics, The Third Xiangya Hospital, Central South University, 138 Tongzipo Road, Changsha, Hunan 410013, P.R. China E-mail: yang_zcr@126.com

Abbreviations: CVB3, coxsackievirus B3; PI3K, phosphatidylinositol 3-kinase; PKB, protein kinase B; mTOR, mammalian target of rapamycin; LAMP-1, lysosomal-associated membrane protein 1; CPE, cytopathic effect; VMC, viral myocarditis

Key words: autophagy, mammalian target of rapamycin, coxsackievirus B3, phosphatidylinositol 3-kinase, viral myocarditis

\section{Introduction}

Viral myocarditis (VMC) is defined as inflammation of the heart muscles. It is a common illness that can lead to severe consequences even death in infants and young adults $(1,2)$. In a recent multicenter analysis of 624 patients with histologically confirmed myocarditis, the presence of various viral genomes was confirmed in 239 biopsy samples (38\%) (3). Among the identified viral genomes, adenovirus, enterovirus and cytomegalovirus were the most common groups. In particular, coxsackievirus B is consistently among the most common. Coxsackieviruses are members of the Picornaviridae family, enterovirus genus. Coxsackievirus $\mathrm{B}$, particularly coxsackievirus B3 (CVB3), has been recognized as the most common cause of VMC associated with heart failure in infants, children and young adults (4). CVB3 infects cells via the coxsackievirus and adenovirus receptor (CAR). The entering of CVB3 induces a direct cytopathic effect (CPE) and even cell death in host cells. It also provokes an immune response. Both of these effects play an important role in the pathogenesis of VMC $(1,5)$. The immune response has a double effect on host cells, and the balance between these positive and negative effects may ultimately determine the course of disease after CVB3 infection $(1,6)$. To date, several pathogenic mechanisms causing tissue injury and fibrosis have been unraveled in CVB3 infection, including direct CVB3-induced damage to the heart tissue, host-cell inflammatory responses to the viral infection or a mixture of these two, which may synergistically promote cardiotoxicity. CVB3-induced acute myocarditis is most likely the consequence of direct virus-induced myocyte damage followed by the host inflammatory response that is associated with persistent CVB3 infection (7). The possible pathogenic mechanisms of CVB3 include activation of caspase-3, phosphorylation and activation of extracellular signal-regulated kinase (ERK)1/2, activation of protein kinase B/Akt (PKB/Akt) and severe endoplasmic reticulum (ER) stress (8-11).

Autophagy is a significant cellular catabolic process in which long-lived proteins and damaged organelles are degraded in lysosomes. Autophagy is a tightly regulated process and defects in autophagy are associated with many human diseases, including cancer, myopathy and neurodegeneration $(12,13)$. Autophagy has also been implicated in the clearance of pathogens and antigen presentation (14-16). In recent years, mounting evidence indicates that autophagy is involved in the process of CVB3-related 
VMC (17,18). CVB3 induces the formation of double-membrane vesicles (autophagosomes) and the conversion of non-modified microtubule-associated protein light chain 3 (LC3)-I to lipidated LC3-II elevated in vitro (19-21). In a model of 3-week-old BALB/c mice with CVB3 infection, electron microscopic observation showed that autophagosome-like vesicles were induced in the cardiac myocytes of mice at 3,5, and 7 days after CVB3 infection. LC3-I and LC3-II were also significantly increased in the myocardium and the cardiac myocytes extracted from the ventricles of mice infected with CVB3. Moreover, viral protein synthesis was significantly decreased in primary cardiac myocytes following treatment with 3-methyladenine, an inhibitor of autophagy (20). CVB3 may exploit the autophagic response to promote viral replication (22) via inhibiting the fusion of autophagosomes with lysosomes to provide sufficient membrane structures for viral RNA replication $(23,24)$.

Mammalian target of rapamycin (mTOR) is described as a key homeostatic regulator of cell growth, proliferation, and survival as well as metabolism by upregulating protein, lipid synthesis and inhibiting excessive autophagy. mTOR is closely related with autophagy induced by viral infection. Sindbis virus and avian influenza viruses can induce autophagy by suppressing mTOR signaling $(25,26)$. Vesicular stomatitis virus induces autophagy by regulating the phosphatidylinositol 3-kinase (PI3K)/Akt signaling pathway (27), and hepatitis $\mathrm{C}$ virus induces autophagy by inactivating the Akt/ TSC/mTOR pathway based on ER stress (28). Avibirnavirus VP2 can induce autophagy through Akt/mTOR pathway inactivation mediated by the HSP90AA1-VP2 complex (29). The function of mTOR is primarily mediated by mTOR complex 1 (mTORC1) and mTORC2. The initiation of autophagy requires the Unc-51-like kinase (ULK) complex and is ultimately regulated by mTORC1. Under fed conditions, the ULK complex is bound to mTORC1, whereas nutrient depletion results in dissociation of the ULK complex from mTORC1. In the unbound state, the ULK complex induces autophagy (30). Recent studies have also established mTORC2 as an independent positive regulator of autophagy $(31,32)$.

Although the number of autophagosomes increases after CVB3 infection, the exact mechanism is unclear. Earlier studies suggested that CVB3 may directly or indirectly induce autophagy via the AMPK/MEK/ERK and Ras/Raf/MEK/ERK signaling pathways in host cells $(20,33)$. In mammalian cells, the PI3K/Akt/mTOR signaling pathway is the primary pathway that regulates autophagy when cells are exposed to certain conditions, such as starvation, oxidative stress, infection and tumor suppression (34). Our previous study demonstrated that the PI3K/Akt/mTOR signaling pathway is associated with apoptosis in CVB3-infected cells $(8,35,36)$. Whether the PI3K/Akt/mTOR signaling pathway is involved in the process of autophagy in CVB3-infected cells is unknown. The present study seeks to examine whether the PI3K/Akt/mTOR signaling pathway participates in the autophagic process after CVB3 infection in HeLa cells.

\section{Materials and methods}

Antibodies and chemical reagents. Polyclonal antibody against LC3-I/II (Sigma-Aldrich, St. Louis, MO, USA), antibody against $\beta$-actin and SQSTM1 rabbit polyclonal antibody/p62 (both from ProteinTech Group, Inc., Chicago, IL, USA) were used at a dilution of 1:1,000. Monoclonal anti-enterovirus antibody (Dako, Carpinteria, CA, USA) was used at a dilution of 1:100. Goat anti-mouse IgG horseradish peroxidase-conjugated secondary antibody and goat anti-rabbit IgG horseradish peroxidase-conjugated secondary antibody (both from CWBIO, Beijing, China) were used at a dilution of 1:2,000. Rapamycin, ZSTK474, MK2206, and chloroquine phosphate were purchased from Selleck Chemicals (Houston, TX, USA).

Cell culture and virus propagation. HeLa cells were obtained from the Institute of Oncology, Central South University, China. The stably transfected cell lines containing the plasmid pcDNA3.1-myc-HisA(-)-Akt1 or the empty vector alone were previously established by our team (35) and kept in our laboratory. Cells were grown in Dulbecco's modified Eagle's medium (DMEM; Hyclone/GE Healthcare Life Sciences, Logan, UT, USA) supplemented with $10 \%$ fetal bovine serum (FBS) and penicillin-streptomycin (both from Gibco Life Technologies, Carlsbad, CA, USA), and incubated at $37^{\circ} \mathrm{C}$ in a humidified incubator with $5 \% \mathrm{CO}_{2}$. CVB3 Nancy strain was obtained from the Shanghai Jiao Tong University School of Medicine and stored at $-80^{\circ} \mathrm{C}$ in our laboratory. CVB3 was propagated in HeLa cells in DMEM supplemented with $2 \%$ FBS, and the virus titer was routinely determined by plaque assay.

Virus infection. HeLa cells were infected with CVB3 at a multiplicity of infection (MOI) of 10. Then, the cells were washed with PBS and replenished with fresh DMEM containing 10\% FBS. DMEM containing 2\% FBS was used for the sham group. Cells were harvested at different times of infection for the next experiments. For inhibition experiments, HeLa cells were pre-treated with the inhibitors (dissolved in DMEM containing 10\% FBS) for $2 \mathrm{~h}$ before viral infection, and DMEM containing 10\% FBS for the sham group. Then, the cells were washed with PBS and infected with CVB3 for $1 \mathrm{~h}$, and fresh DMEM containing 2\% FBS for the sham group.

Cell transfection. HeLa cells at $80 \%$ confluency were transfected with pEGFP-LC3 or an empty vector (pEGFP-C3). Four milligrams of expression plasmid combined with $10 \mu \mathrm{l}$ Lipofectamine 2000 reagent (Invitrogen Life Technologies, Carlsbad, CA, USA) was added to the cells according to the manufacturer's instructions. At 5 h post-transfection, the cells were refreshed with DMEM containing $10 \%$ FBS. Twenty-four hours later, the cells were seeded in 6-well plates for the next experiments.

Immunofluorescence assay. HeLa cells transfected with plasmid pEGFP-LC3 or pEGFP-C3 were seeded on cover glasses and exposed to CVB3 or not. Cells were washed thrice with cold PBS. The different groups were fixed in $4 \%$ paraformaldehyde for $10 \mathrm{~min}$ at room temperature, permeabilized and then blocked with $5 \%$ bovine serum albumin in $0.3 \%$ Triton X-100 (diluted in PBS) for $2 \mathrm{~h}$. The samples were incubated with anti-lysosomal-associated membrane protein 1 (LAMP-1) (1:100) overnight at $4^{\circ} \mathrm{C}$ and then washed thrice with $0.3 \%$ Triton $\mathrm{X}-100$ (diluted with deionized water) and incubated for $60 \mathrm{~min}$ with HRP-conjugated goat anti-mouse IgG. After three washes, the 
Table I. Primer sequences.

\begin{tabular}{ll} 
Gene & \multicolumn{2}{c}{ Primer sequences } \\
\hline CVB3 & F: 5'-TGGTGGGCTATGGAGTATGG-3' \\
& R: 5'-CACTGGATGGGGTGTTGTCT-3' \\
$\beta$-actin & F: 5'-CTAAGGCCAACCGTGAAAAGATGAC-3' \\
& R: 5'-TGGGTACATGGTGGTGCCACCAGAC-3'
\end{tabular}

This table contains the sequences of primers used in the process of semi-quantitative PCR. CVB3, coxsackievirus B3.

cells were stained with DAPI $(1: 1,000)$ for 5 min and washed again. Afterwards, cover slips were mounted onto glass microscopic slides, and the samples were observed under an Olympus microscope equipped with a MetaMorph image acquisition system (DP2-BSW software) (both from Olympus Corp., Tokyo, Japan).

Western blot analysis. HeLa cells exposed to various conditions were washed twice with ice-cold PBS and then lysed with RIPA lysis buffer (CWBIO) containing $0.1 \%$ phenylmethylsulfonyl fluoride (PMSF; CWBIO). Afterwards, the lysates were sonicated and centrifuged at $13,000 \mathrm{rpm}$ for $15 \mathrm{~min}$ at $4^{\circ} \mathrm{C}$. The samples were subsequently boiled and denatured. The protein concentration was determined by the Enhanced BCA protein Assay kit (Beyotime Institute of Biotechnology, Shanghai, China). Briefly, $30 \mu \mathrm{g}$ of cell protein samples was subjected to $10-12 \%$ SDS polyacrylamide gel electrophoresis and transferred to 0.45-mm PVDF membranes (EMD Millipore, Billerica, MA, USA). After blocking with 5\% non-fat milk for $3 \mathrm{~h}$ (room temperature), the membranes were incubated with the primary antibody at $4^{\circ} \mathrm{C}$ overnight. After washing with PBST thrice, the membranes were incubated with horseradish peroxidase-conjugated secondary antibodies (CWBIO) for 60 min followed by washing. Finally, protein bands were detected with X-ray film, and protein expression was quantitated by densitometric analysis using NIH Image J.

Confocal microscopy. To determine whether autophagosomes in CVB3-infected HeLa cells fused with lysosomes, cells were transiently transfected with the pEGFP-LC3 plasmid using Lipofectamine 2000 (Invitrogen Life Technologies). After $24 \mathrm{~h}$ of transfection, the cells were infected with CVB3 or not and then incubated with anti-LAMP-1 overnight (see details in 'Immunofluorescence assay'). We observed the co-localization of LAMP-1 and GFP-LC3 under a Leica SP2 AOBS confocal fluorescence microscope. Autophagosomes can be distinguished from amphisomes or autolysosomes as follows: green vesicles [GFP-LC3 ${ }^{+}$LAMP-1-negative (LAMP-1)] are autophagosomes; yellow vesicles [GFP-LC3 ${ }^{+}$ LAMP-1-positive (LAMP-1 ${ }^{+}$)] are amphisomes; and red vesicles [GFP-LC3-negative (pEGFP-LC3') LAMP-1 ${ }^{+}$] cannot be categorized solely on the basis of LAMP-1 expression, as they may be endosomes, lysosomes, or autolysosomes.

Transmission electron microscopy (TEM). For ultrastructural analysis, HeLa cells were mock infected or infected
Table II. PCR conditions.

Genes Conditions

CVB3

$95^{\circ} \mathrm{C}$ for $2 \mathrm{~min}, 30 \mathrm{sec} ; 94^{\circ} \mathrm{C}$ for $40 \mathrm{sec} ; 59^{\circ} \mathrm{C}$ for $40 \mathrm{sec}, 72^{\circ} \mathrm{C}$ for $40 \mathrm{sec}, 35$ cycles; $72^{\circ} \mathrm{C}$ for $5 \mathrm{~min}$

This table contains the PCR conditions used in the process of semi-quantitative PCR. CVB3, coxsackievirus B3.

with CVB3 at MOI of 10 for $1 \mathrm{~h}$. Cells were collected at 24 hours post-inoculation (h.p.i.) and centrifuged at 1,200 rpm for $10 \mathrm{~min}$ at room temperature. Cells were then fixed in $2.5 \%$ glutaraldehyde in PBS for $10 \mathrm{~min}$ at room temperature. Following three washes in PBS, the cells were post-fixed in $2 \%$ osmium tetroxide for $2 \mathrm{~h}$. Then, the cells were dehydrated in a graded series of acetone washes successively. Then, the cells were soaked in epoxy resin mixed with an equal volume of pure acetone and embedded in Eponate 12 resin for $24 \mathrm{~h}$. Finally, the dyed slices were observed, and images were obtained via TEM (Hitachi H-7500; Hitachi, Ltd., Tokyo, Japan). Approximately 15 cells were counted. The number of double-membrane vesicles for each cell was examined. Autophagosomes were defined as double-membrane vacuoles measuring $0.2-0.5 \mu \mathrm{m}$.

Semi-quantitative PCR. HeLa cells either untreated or treated with different inhibitors were harvested at 24 h.p.i. mRNA was extracted following the method of the RNA extraction kit (Omega Bio-Tek, Inc., Norcross, GA, USA). Next, mRNA was reverse transcribed into cDNA following the RevertAid First Strand cDNA Synthesis kit (Thermo Fisher Scientific, Waltham, MA, USA). Finally, PCR amplification was performed. The primer sequences and PCR conditions are provided in Tables I and II [refer to Li et al (36)].

Drug treatment. To identify the autophagic response in HeLa cells infected by CVB3, rapamycin (100 $\mathrm{mM}$ dissolved in DMEM containing 10\% FBS) served as a positive control and $24 \mathrm{~h}$ later, the cells were harvested for western blot analysis. Chloroquine phosphate (inhibition of lysosome function, $40 \mu \mathrm{M}$ ) was used to assess autophagic flux caused by CVB3 infection, and rapamycin (10 nM dissolved in DMEM containing $10 \%$ FBS) was used to inhibit the function of mTOR; $2 \mathrm{~h}$ later, the cells were washed thrice and infected with CVB3. ZSTK474 (50 $\mu \mathrm{M})$ was used at a concentration of $50 \mu \mathrm{M}$ to inhibit PI3K and $2 \mathrm{~h}$ later, the cells were washed thrice and infected or not with CVB3. To suppress the overexpression of Akt1, MK2206 (inhibitor of Akt, $50 \mu \mathrm{M}$, dissolved in DMEM containing 10\% FBS) was used to pre-treat the pcDNA3.1-myc-HisA(-)-Akt1-HeLa cells. After $2 \mathrm{~h}$, the cells were infected and harvested at 24 h.p.i. for western blot analysis.

Statistical analysis. Two-way analysis of variance with multiple comparisons and paired Student's t-tests were performed. Data are presented as the means \pm standard error (SE). A value of $\mathrm{P}<0.05$ was considered to indicate a statistically significant difference. 

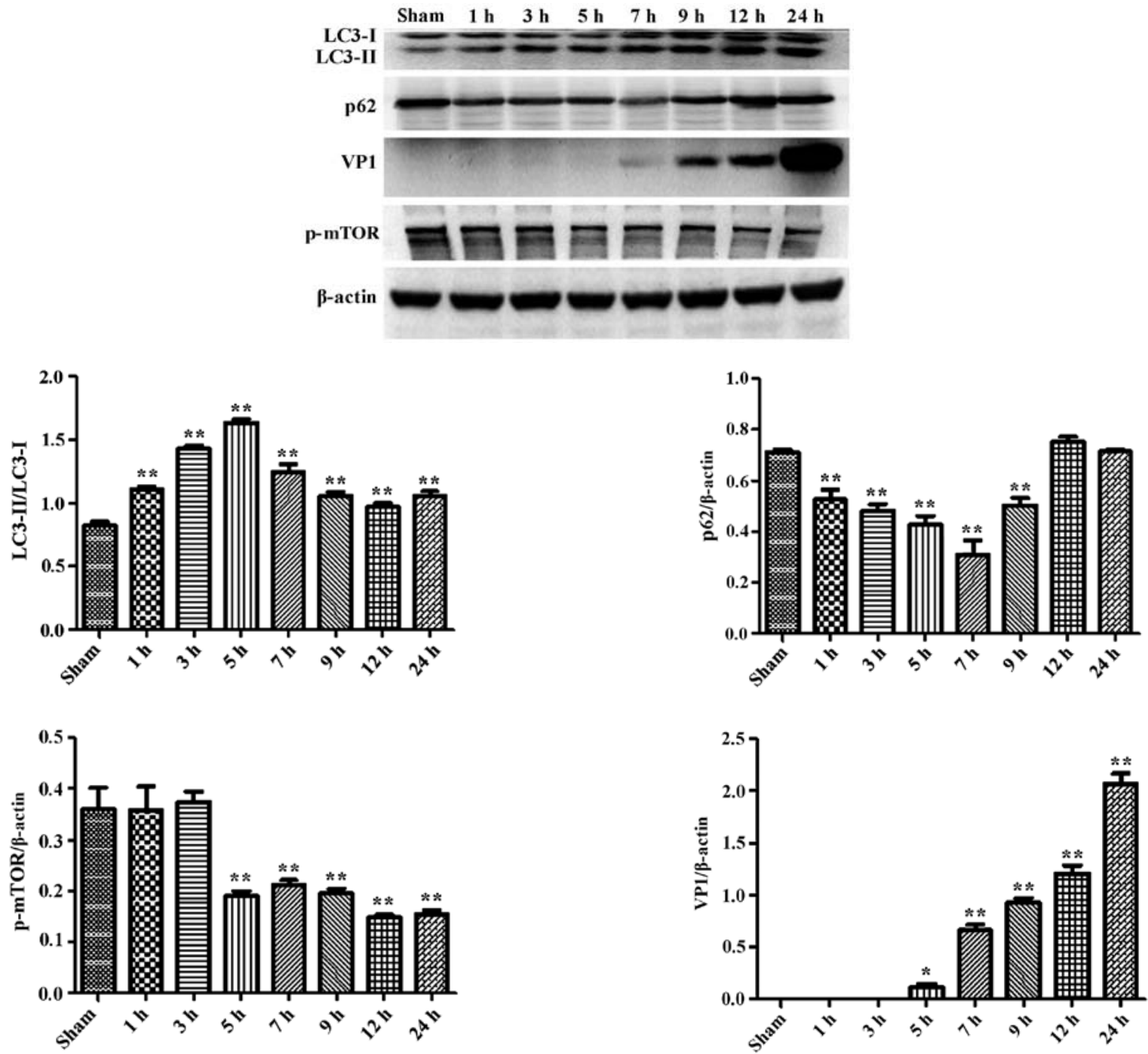

Figure 1. CVB3 induces an autophagic response at different times during CVB3 infection in HeLa cells. HeLa cells were infected with CVB3, cell lysates were collected at 1, 3, 5, 7, 9, 12 and 24 h.p.i., and mock-infected HeLa cells served as the sham control. LC3, p62, p-mTOR and viral capsid protein VP1 were examined by western blot analysis, and these samples were immunoblotted with an antibody to $\beta$-actin to illustrate equal protein loading. Compared with the sham group, the LC3-II/LC3-I ratio increased at 1 h.p.i. then peaked at 5 h.p.i. ( $<<0.05)$. p62 exhibited a decrease at 1, 3, 5, 7 and 9 h.p.i. ( $p<0.01$ ). No changes were noted at 12 and 24 h.p.i. compared with the sham group. p-mTOR showed a decrease after 5 h.p.i. $(\mathrm{p}<0.05)$, which was more obvious at 12 and 24 h.p.i. VP1 increased as the time of infection increased. ${ }^{*} \mathrm{P}<0.05$, compared with the sham group; ${ }^{* *} \mathrm{p}<0.01$, compared with the sham group. CVB3, coxsackievirus B3; h.p.i., hours post-inoculation; LC3, light chain 3; mTOR, mammalian target of rapamycin.

\section{Results}

CVB3 induces an autophagic response at different times during CVB3 infection in HeLa cells. During the process of autophagosome formation, the conversion of cytosolic LC3-I into the lipidated form LC3-II is pivotal. Thus, the processing from LC3-I to LC3-II represents the appearance of autophagosomes or autophagic response. The membrane-bound form of LC3 (LC3-II) is considered as the most appropriate marker of autophagosomes in mammalian cells. The protein p62 is considered as a marker for autophagy-mediated protein degradation or autophagic flux (37); it functions as an autophagy receptor targeting ubiquitinated proteins to autophagosomes for degradation. To characterize the autophagic reaction and the change of viral replication during CVB3 infection, HeLa cells were infected with CVB3. Cell lysates were collected at 1, 3, 5, 7, 9, 12 and 24 h.p.i. Mock-infected HeLa cells served as the sham control. We examined LC3, p62, p-mTOR and the viral capsid protein VP1 by western blot analysis at different times during CVB3 infection (Fig. 1). The LC3-II/LC3-I ratio increased at 1 h.p.i. and reached its peak at 5 h.p.i. $(\mathrm{p}<0.05)$. p62 showed a decrease at 1,3, 5, 7 and 9 h.p.i. $(\mathrm{p}<0.05)$. No changes were noted at 12 and 24 h.p.i. compared with the sham group. p-mTOR exhibited a decrease at 5, 7, and 9 h.p.i $(\mathrm{p}<0.05)$, which was more obvious at 12 and 24 h.p.i. We detected obvious VP1 expression 5 h.p.i. $(\mathrm{p}<0.05)$ which increased as the infected time increased $(\mathrm{p}<0.01)$.

Confirmation of autophagy activation in HeLa cells at 24 h.p.i. Given that LC3 and p-mTOR was altered during CVB3 infection, to further characterize the effects of the mTOR pathway during CVB3 infection, we selected 24 h.p.i. as our research time point to conduct our next study.

Primarily, we identified the autophagic response in HeLa cells infected by CVB3. We determined the activation of autophagy using three criteria. First, we analyzed the lipi- 

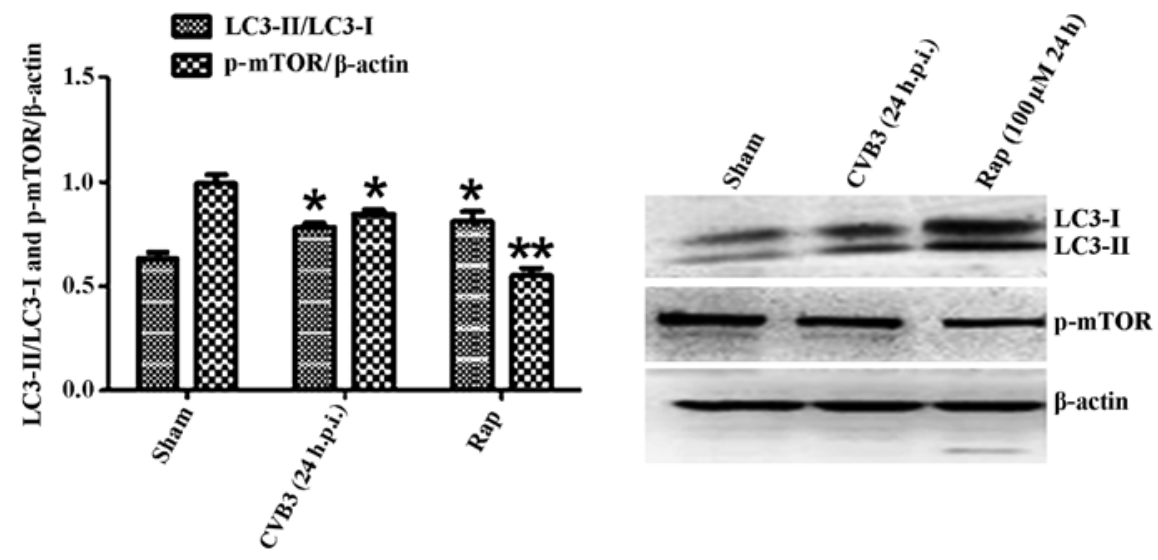

Figure 2. LC3 and p-mTOR-are altered in HeLa cells at 24 h.p.i. HeLa cells were infected with CVB3. DMEM containing $2 \%$ FBS served as the sham control, and rapamycin (Rap) $(100 \mu \mathrm{M})$ served as a positive control. At 24 h.p.i. cells were collected for western blot analysis. Compared with the sham group, the total amount of LC3 (LC3-I, LC3-II) and the ratio of LC3-II/LC3-I were markedly increased in the infected cells at 24 h.p.i. (p<0.05). The expression of p-mTOR was downregulated $(\mathrm{p}<0.05)$. ${ }^{*} \mathrm{p}<0.05$, compared with the sham group; ${ }^{* *} \mathrm{p}<0.01$, compared with the sham group. LC3, light chain 3; mTOR, mammalian target of rapamycin; h.p.i., hours post-inoculation; CVB3, coxsackievirus B3; DMEM, Dulbecco's modified Eagle's medium; FBS, fetal bovine serum.
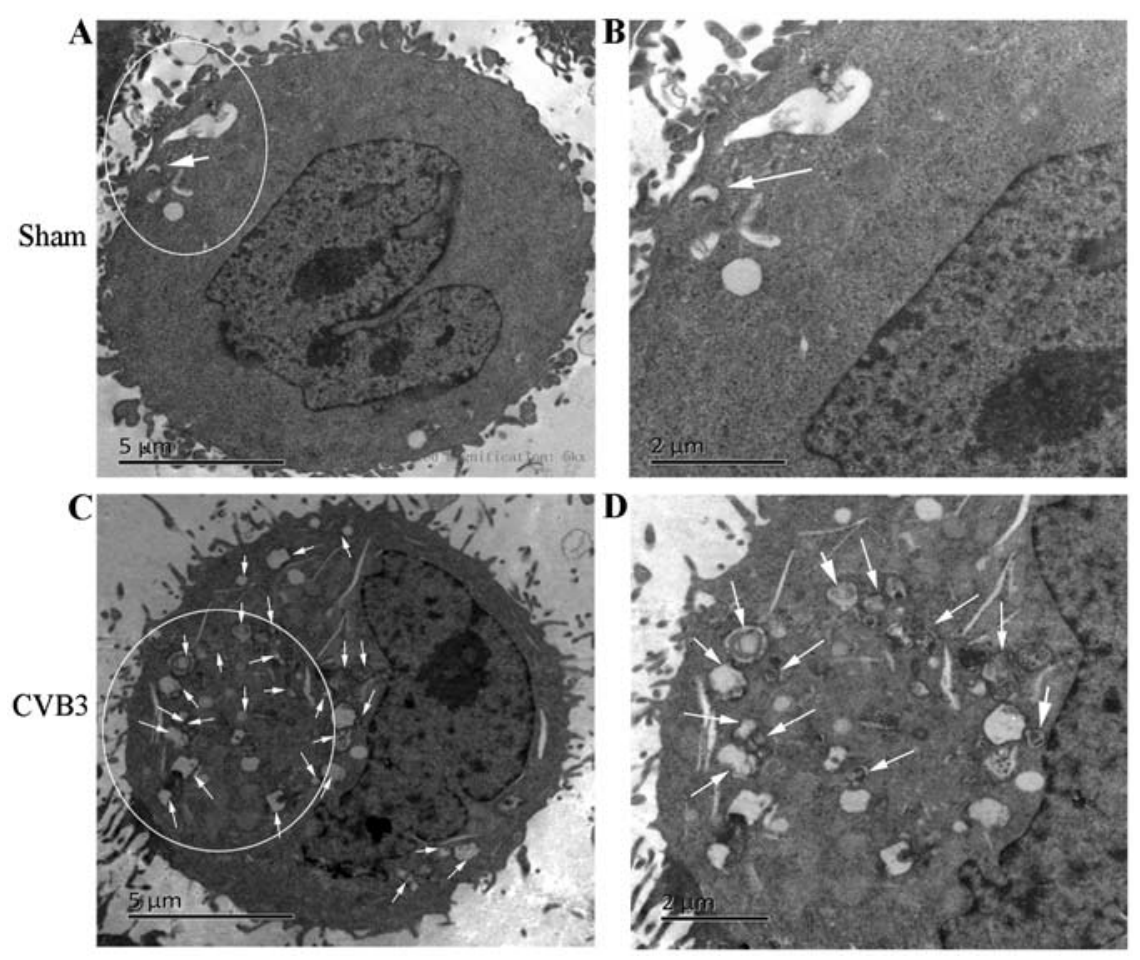

Figure 3. TEM analysis of HeLa cells at 24 h.p.i. HeLa cells were collected at 24 h.p.i. and subjected to electron microscopic observation. Abundant autophagosome-like vesicles with double membranes (0.2-0.5 $\mu \mathrm{m}$ in size) clustered in the CVB3-infected group compared with the control group (white arrows). (A and B) The normal structure in the sham group. (C and D) The changes in CVB3-infected HeLa cells. Arrows indicate representative autophagosomes that would be scored positive in (B), with double membranes 0.2-0.5 $\mu \mathrm{m}$ in size. TEM, transmission electron microscopy; h.p.i., hours post-inoculation; CVB3, coxsackievirus B3.

dated form of the microtubule-associated protein 1 LC3 in the CVB3-infected HeLa cells and mock-infected cells, and rapamycin $(100 \mu \mathrm{M})$ served as a positive control (Fig. 2). The results revealed that the total amount of LC3 (LC3-I, LC3-II) and the ratio of LC3-II/LC3-I were markedly increased in the infected cells at 24 h.p.i. compared with the mock-infected cells $(\mathrm{p}<0.05)$. The expression of $\mathrm{p}-\mathrm{mTOR}$ was decreased $(p<0.05)$. Second, we found that double-membrane autophagosomes, which are prominent features of autophagy (Fig. 3), were increased in the CVB3-infected HeLa cells. Finally, we observed that LC3 puncta formation was increased following CVB3 infection (Fig. 4). We counted 50 transduced cells/ well for quantification of cells with high levels of GFP-LC3 autophagosome vacuoles, with 3 wells/group (represented as the mean \pm SEM). Quantification of $>30$ punctate/cell was defined as a high level of GFP-LC3 autophagosome vacuoles. We observed a statistically significant increase in LC3 puncta formation within the infected pEGFP-LC3-HeLa cells at 24 h.p.i. compared with that in the mock-infected HeLa cells or the CVB3-infected pEGFP-C3-HeLa cells (Fig. 5, 
A

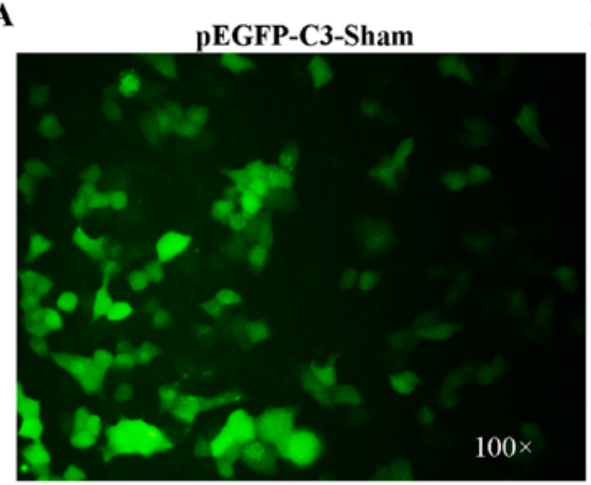

C

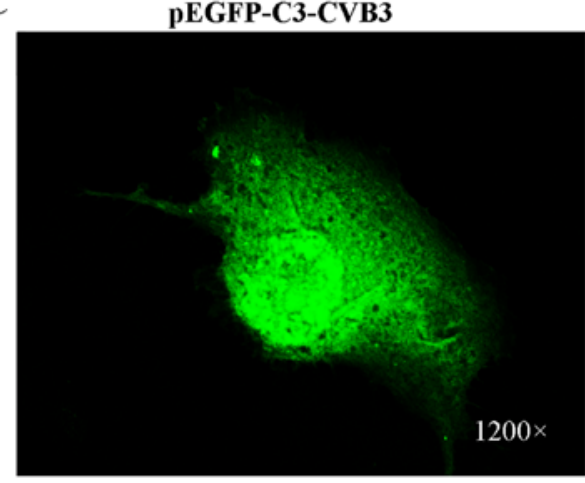

B

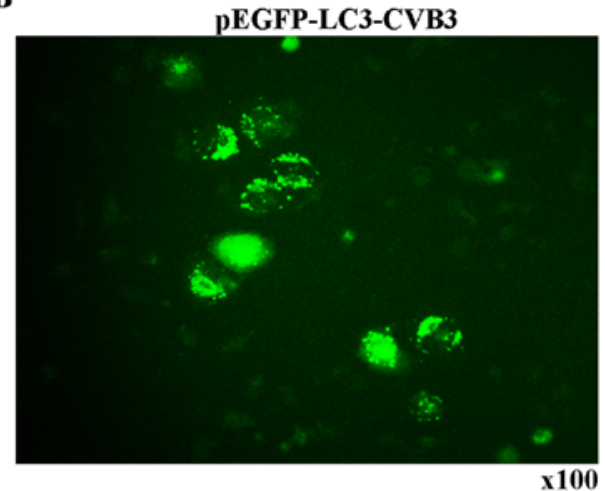

D

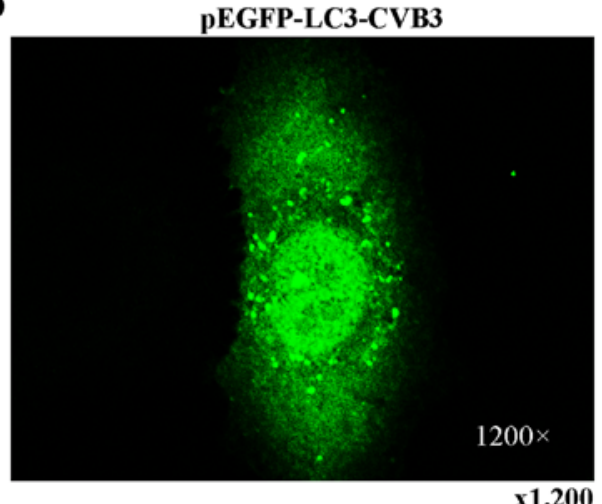

Figure 4. LC3 puncta formation is increased in the HeLa cells at 24 h.p.i. HeLa cells were transfected with plasmid pEGFP-LC3 or the empty vector (pEGFP-C3) using Lipofectamine 2000 (Invitrogen Life Technologies). Twenty-four hours later, fluorescence microscopy demonstrated the successful transduction of cells with pEGFP-LC3 and pEGFP-C3. Then, the cells were seeded in 6-well plates. (B-D) Twenty-four hours later, the cells were infected with CVB3, and (A) mock-infected cells served as the control group. LC3, light chain 3; h.p.i., hours post-inoculation; CVB3, coxsackievirus B3.

$\mathrm{p}<0.05)$. To assess the effects of CVB3 on autophagosome fusion with endosomes/lysosomes, we observed the distribution of LAMP-1, a protein found in endosomes and lysosomes. Autophagosomes can be distinguished from amphisomes or autolysosomes as follows: green vesicles (pEGFP-LC3 ${ }^{+} / \mathrm{LAMP}^{-}{ }^{-}$) are autophagosomes; yellow vesicles (pEGFP-LC3 ${ }^{+} / \mathrm{LAMP}^{+}{ }^{+}$) are amphisomes; and red vesicles ( $\mathrm{pEGFP}-\mathrm{LC} 3^{-} / \mathrm{LAMP}^{-}{ }^{+}$) cannot be categorized solely on the basis of LAMP-1 expression, as they may be endosomes, lysosomes, or autolysosomes (pEGFP-LC3-II is degraded in autolysosomes), In addition, GFP fluorescence is quenched in an acidic environment (38). In our research, we observed yellow dots, which suggested the co-localization of GFP-LC3 and LAMP-1 (Fig. 6).

Our findings revealed that CVB3 triggered autophagic response in HeLa cells, in accordance with previous studies $(19,20)$.

Rapamycin aggravates the autophagic reaction induced by CVB3 infection. To further elucidate the impact of mTOR during the course of CVB3 infection, HeLa cells were treated with rapamycin (10 $\mathrm{nM}$ dissolved in DMEM containing $10 \%$ FBS) for $2 \mathrm{~h}$. Then, the cells were washed thrice and infected with CVB3 (rapamycin group). In the CVB3 group, HeLa cells were incubated with DMEM containing 10\% FBS for $2 \mathrm{~h}$ and then infected with CVB3. The control group was incubated with DMEM containing 10\% FBS. Two hours later, the medium was refreshed with DMEM containing 2\% FBS. At 24 h.p.i.,

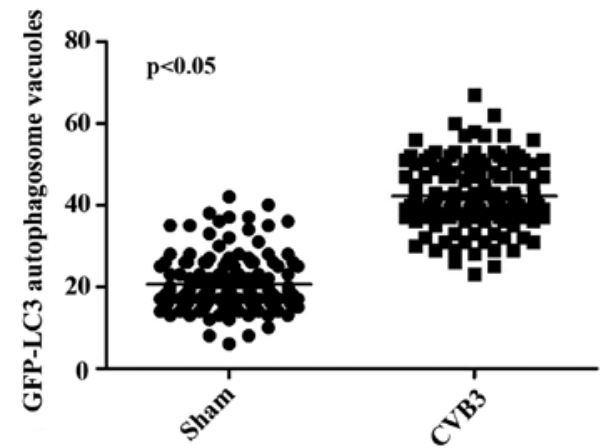

Figure 5. Quantification of GFP-LC3 dots in the sham group and infected group. LC3, light chain 3 .

the cells were collected for western blot analysis (Fig. 7). The LC3-II/LC3-I ratio was increased in the CVB3 group and the rapamycin group $(\mathrm{p}<0.01)$, and $\mathrm{p}-\mathrm{mTOR}$ was decreased in the CVB3 group $(\mathrm{p}<0.05)$ and rapamycin group $(\mathrm{p}<0.01)$, compared with the control group. When comparing the virus-infected condition with the rapamycin + virus-infected condition, we found that pre-treatment of the cells with rapamycin aggravated the autophagy induced by CVB3 infection $(\mathrm{p}<0.05)$. Furthermore, we used chloroquine phosphate (inhibition of lysosome function, $40 \mu \mathrm{M}, 2 \mathrm{~h}$ before infection) to assess autophagic flux caused by CVB3 infection (CQ group) and monitored p62. The LC3-II/LC3-I ratio and p62 were 

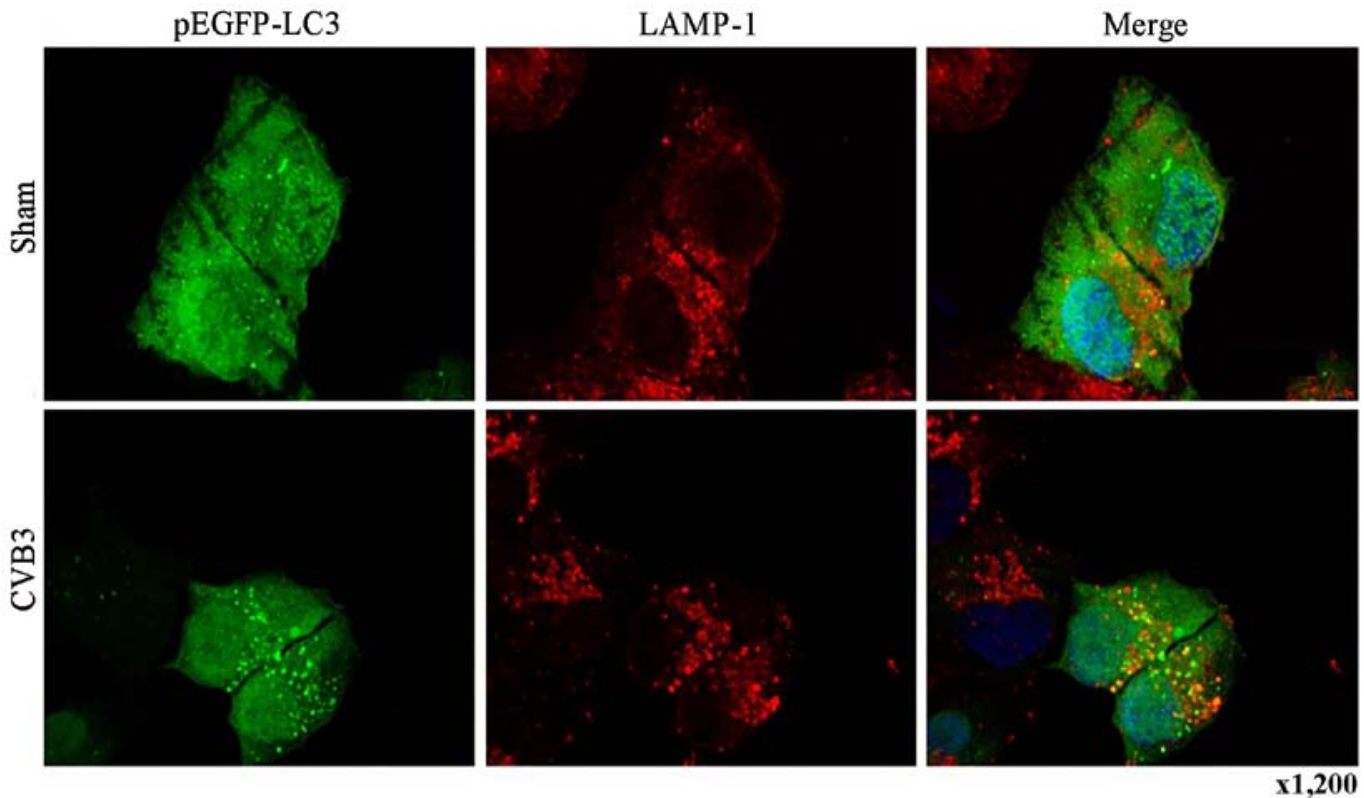

Figure 6. Co-localization of GFP-LC3 and LAMP-1 in HeLa cells at 24 h.p.i. HeLa cells were transfected with the pEGFP-LC3 plasmid for $24 \mathrm{~h}$ followed by CVB3 infection. Twenty-four hours later, the transfected cells were stained with an antibody specific for the endosomal/lysosomal marker LAMP-1, and the co-localization of GFP-LC3 and LAMP-1 vesicles was examined by confocal microscopy. Single-color fluorescence images of pEGFP-LC3 and LAMP-1 are presented in the 1st and 2nd columns, and a merged view of these two proteins is shown in the 3rd column. A yellow signal signifies co-localization of pEGFP-LC3 and LAMP-1. LC3, light chain 3; LAMP-1, lysosomal-associated membrane protein 1; h.p.i., hours post-inoculation; CVB3, coxsackievirus B3.
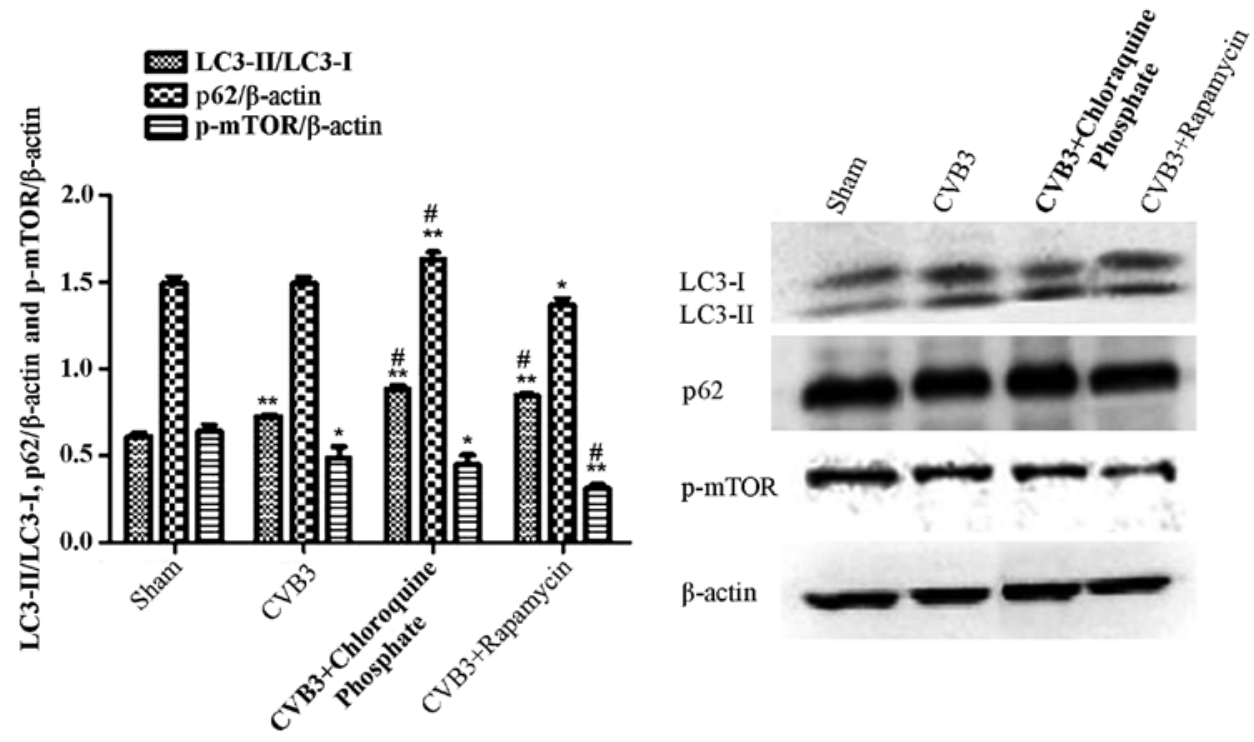

Figure 7. Rapamycin aggravates the autophagic reaction induced by CVB3 infection. HeLa cells were treated with $10 \mathrm{nM}$ rapamycin and chloroquine phosphate (CQ group) for $2 \mathrm{~h}$. The cells were washed thrice and infected with CVB3. In the CVB3 group, HeLa cells were incubated with DMEM containing 10\% FBS for $2 \mathrm{~h}$ and infected with CVB3. The sham group was incubated with DMEM containing 10\% FBS. Two hours later, the medium was refreshed with DMEM containing 2\% FBS. At 24 h.p.i., cells were collected for western blot analysis. The LC3-II/LC3-I ratio was increased in the CVB3 and rapamycin group $(\mathrm{p}<0.01)$, and $\mathrm{p}-\mathrm{mTOR}$ was decreased in the CVB3 group $(\mathrm{p}<0.05)$ and rapamycin group $(\mathrm{p}<0.01)$ compared with the sham group. The changes in the LC3-II/LC3-I ratio and p-mTOR were more obvious in the Rap group compared with the CVB3 group ( $p<0.05)$. The LC3-II/LC3-I ratio and p62 was increased in the CQ group compared with the CVB3 group $(\mathrm{p}<0.05)$. " $\mathrm{p}<0.05$, compared with the sham group; ${ }^{* *} \mathrm{p}<0.01$, compared with the sham group; ${ }^{*} \mathrm{p}<0.05$, compared with the CVB3 group. CVB3, coxsackievirus B3; DMEM, Dulbecco's modified Eagle's medium; FBS, fetal bovine serum; h.p.i., hours post-inoculation; LC3, light chain 3; mTOR, mammalian target of rapamycin.

increased more obviously in the CQ group compared with the CVB3 group $(\mathrm{p}<0.05)$.

ZSTK474 alleviates the autophagic response caused by CVB3 infection in HeLa cells. To further elucidate whether $\mathrm{PI} 3 \mathrm{~K}$ is involved in CVB3-induced autophagy, we used
ZSTK474, a novel PI3KC1 inhibitor, to assess the effects of PI3KC1 on autophagy during CVB3 infection. HeLa cells were pre-treated with ZSTK474 $(50 \mu \mathrm{M}, 2 \mathrm{~h})$, and the cells were infected with CVB3 or not. Cell lysates were collected at 24 h.p.i. The expression levels of LC3 and phosphorylated Akt1 (p-Akt1) were determined by western blot anal- 


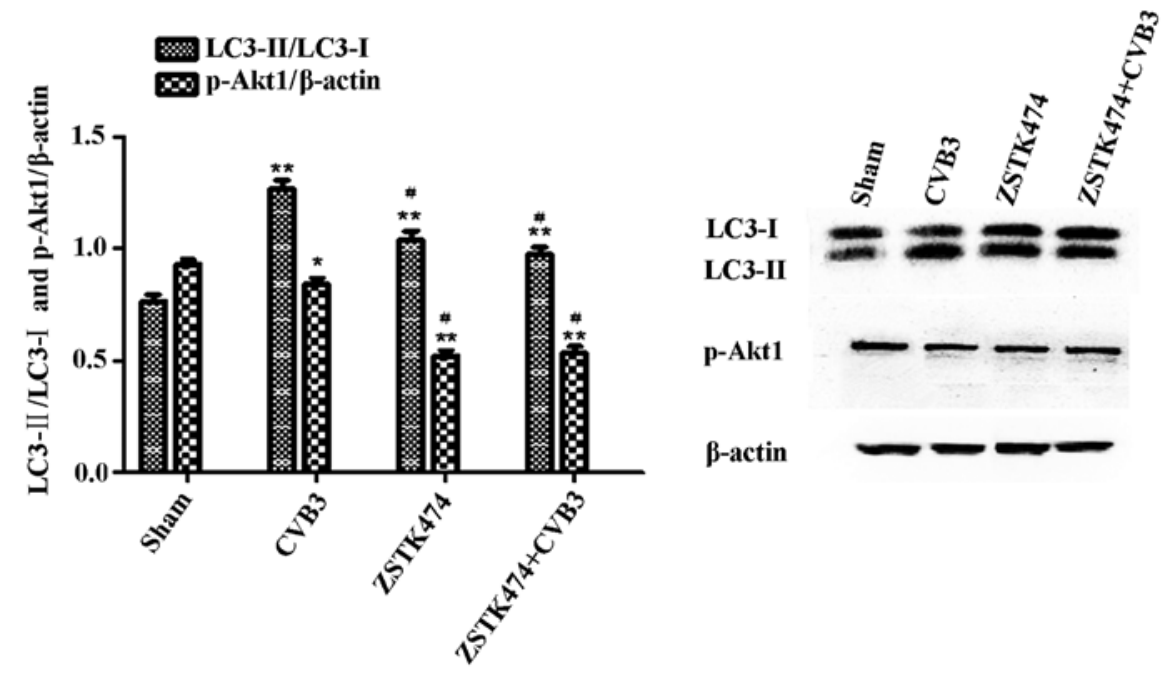

Figure 8. Inhibition of PI3K with ZSTK474 alleviates the autophagic reaction caused by CVB3 infection. HeLa cells were pre-treated with ZSTK474 (50 $\mu \mathrm{M}$, $2 \mathrm{~h}$ ) and then infected with CVB3 (ZSTK4747 + CVB3 group) or not (CVB3 group). Mock-infected cells served as the sham control. Cell lysates were collected at 24 h.p.i., and the expression of LC3 and p-Akt1 was determined by western blot analysis. Compared with the sham group, p-Akt1 was decreased in the CVB3 group $(\mathrm{p}<0.05)$ and the ZSTK474 group $(\mathrm{p}<0.01)$. The LC3-II/LC3-I ratio was increased $(\mathrm{p}<0.01)$. The LC3-II/LC3-I ratio was increased in the CVB3 group compared with the ZSTK474 + CVB3 group ( $<<0.05$ ). ${ }^{*} \mathrm{p}<0.05$, compared with the sham group; ${ }^{* *} \mathrm{p}<0.01$, compared with the sham group; ${ }^{*} \mathrm{p}<0.05$, compared with the CVB3 group. PI3K, phosphatidylinositol 3-kinase; CVB3, coxsackievirus B3; h.p.i., hours post-inoculation; LC3, light chain 3; p-Akt1, phosphorylated Akt1.
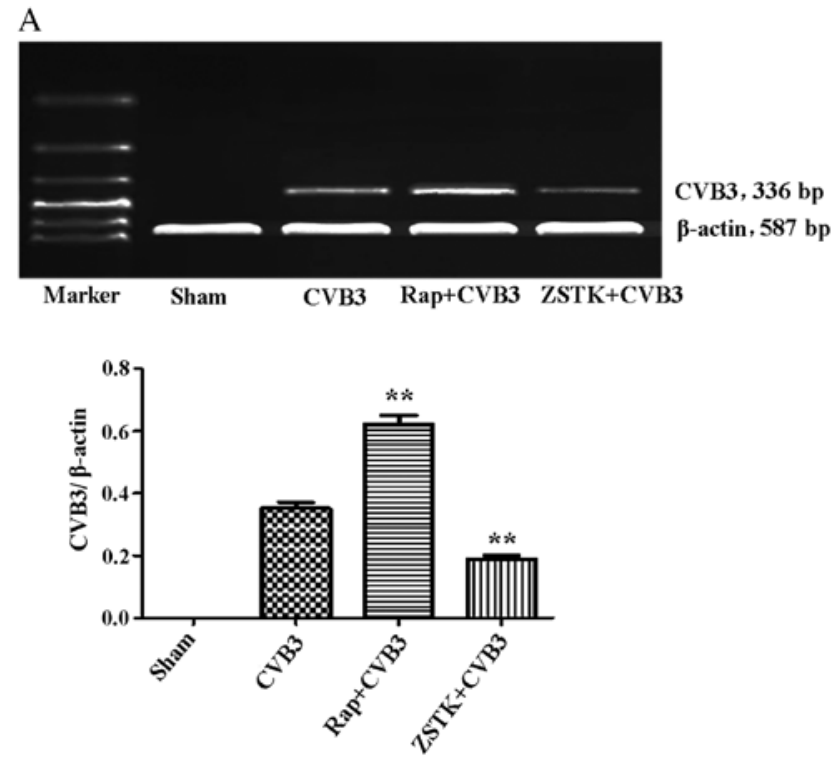
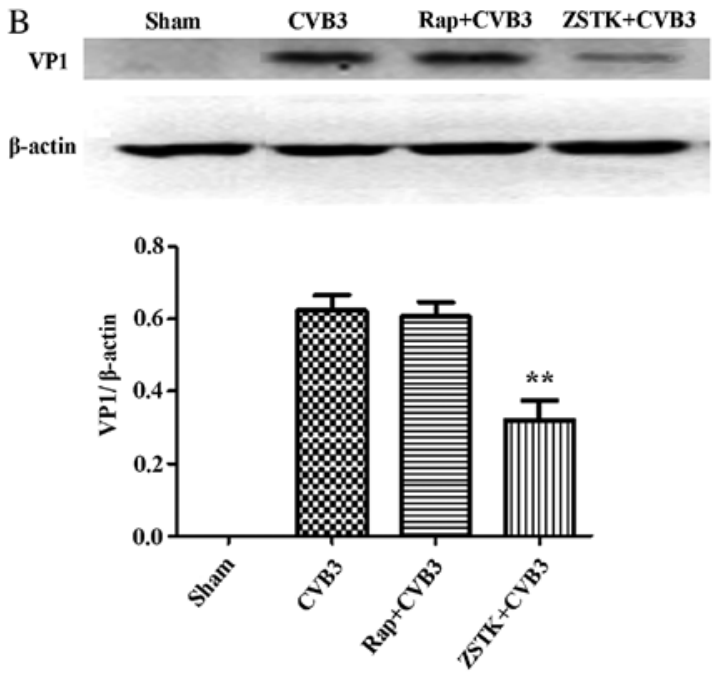

Figure 9. Rapamycin and ZSTK474 affect viral replication. HeLa cells pre-treated with rapamycin (Rap) (10 nM) and ZSTK474 (ZSTK) (50 $\mu$ M) were infected with CVB3, and we examined (A) mRNA and (B) viral capsid protein VP1 by semi-quantitative PCR and western blot analysis, respectively. Compared with the CVB3 group, CVB3 mRNA and VP1 expression were decreased in the ZSTK474 group (p<0.01). Rapamycin stimulated viral mRNA synthesis caused by viral infection $(\mathrm{p}<0.01) .{ }^{* *} \mathrm{p}<0.01$, compared with the sham group. CVB3, coxsackievirus B3.

ysis (Fig. 8). The results showed that p-Akt1 was decreased in the CVB3 infection group $(\mathrm{p}<0.05)$ and the ZSTK474 treatment group $(\mathrm{p}<0.01)$ accompanied by a significant increase in the LC3-II/LC3-I ratio ( $\mathrm{p}<0.01)$. The LC3-II/LC3-I ratio in the CVB3-infected + ZSTK474 group was reduced compared with that in the CVB3-infected group $(\mathrm{p}<0.05)$.

Rapamycin and ZSTK474 affect viral replication. To further characterize the change in viral replication when this pathway was blocked, HeLa cells pre-treated with rapamycin $(10 \mathrm{nM})$ and ZSTK474 $(50 \mu \mathrm{M})$ were infected with CVB3, and we examined mRNA (Fig. 9A) and viral capsid protein
VP1 (Fig. 9B) by semi-quantitative PCR and western blot analysis, respectively. ZSTK474 inhibited viral mRNA synthesis and blocked viral protein VP1 expression caused by CVB3 infection $(\mathrm{p}<0.01)$, whereas rapamycin stimulated viral mRNA synthesis $(\mathrm{p}<0.01)$. However, the viral protein VP1 did not mirror the mRNA changes in the rapamycin group.

Inhibition of Aktl aggravates the autophagic response caused by CVB3 infection in Aktl-overexpressing cells. To clarify whether the changes in Akt1 affect the autophagic reaction in CVB3-induced autophagy, we examined the autophagy activation in cell lines containing the plasmid 

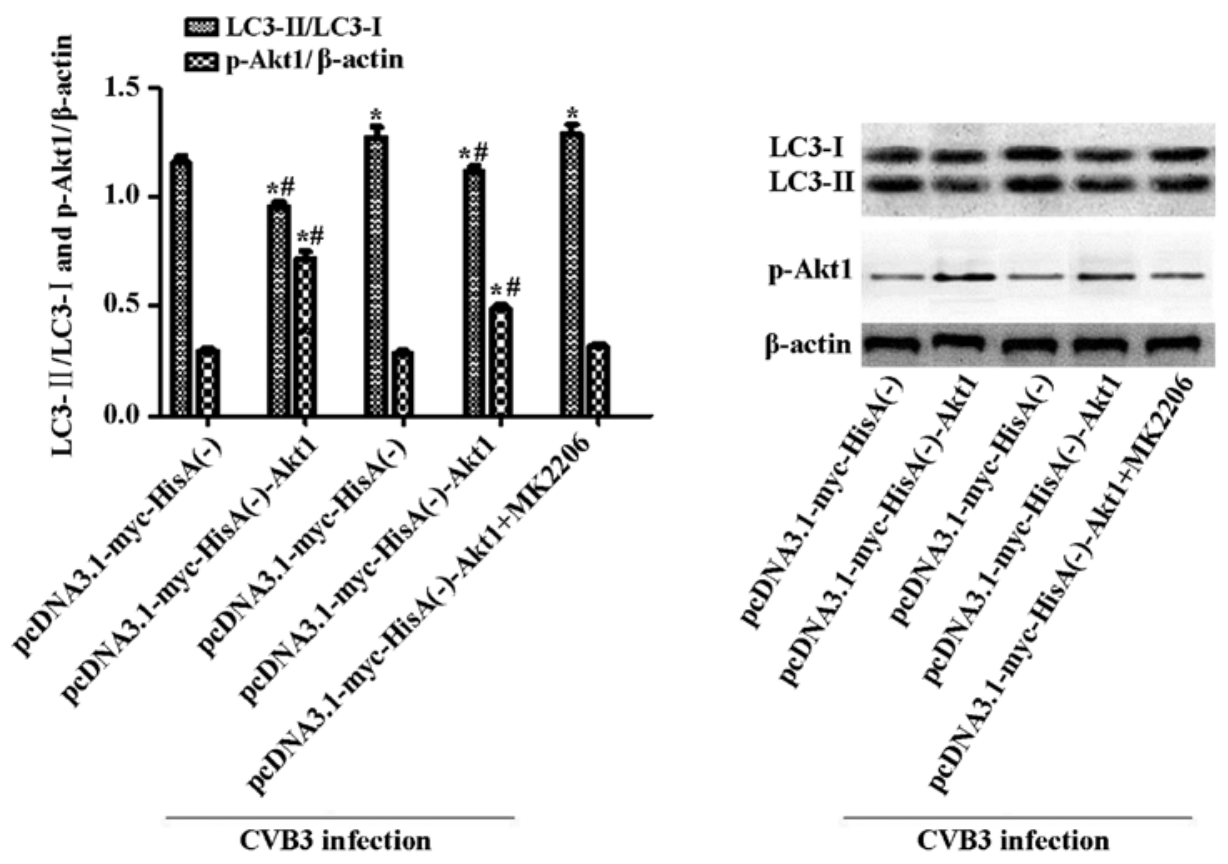

Figure 10. Inhibition of Akt1 aggravates the autophagic response caused by CVB3 infection in Akt1-overexpressing cells. Cells overexpressing Akt1 or harboring empty vector alone were infected with CVB3 or not. Cells were collected for western blot analysis at 24 h.p.i. To suppress the overexpression of Akt1, MK2206 $(50 \mu \mathrm{M})$ was used to pre-treat the pcDNA3.1-myc-HisA(-)-Akt1 HeLa cells. Two hours later, the cells were infected and harvested at 24 h.p.i., and mock-infected cells served as the sham control. In Akt1-overexpressing cell lines, CVB3 infection increased the LC3-II/LC3-I ratio ( $<<0.05$ ). The ratio was more significantly increased when the infected cells were pre-treated with MK2206 ( $<<0.05)$. Under uninfected conditions, the LC3-II/LC3-I ratio was higher in Akt1-overexpressing cells compared with the empty vector cells $(\mathrm{p}<0.05)$. ${ }^{*} \mathrm{p}<0.05$, compared with the pcDNA3.1-myc-HisA(-) group; ${ }^{\# p}<0.05$, compared with the pcDNA3.1-myc-HisA(-)-Akt1+CVB3 group. CVB3, coxsackievirus B3; h.p.i., hours post-inoculation; LC3, light chain 3.

pcDNA3.1-myc-HisA(-)-Akt1 or the empty vector alone. The establishment of stable cell lines overexpressing Akt1 was described in our previous study (35). The cell lines overexpressing Akt1 or the containing empty vector alone were infected with CVB3. The cells were collected for western blot analysis at 24 h.p.i. To suppress the overexpression of Akt1, we used MK2206 (inhibitor of Akt, $50 \mu \mathrm{M}$, dissolved in DMEM containing 10\% FBS) to pre-treat the pcDNA3.1-myc-HisA(-)-Akt1-HeLa cells. Two hours later, the cells were infected and harvested at 24 h.p.i. LC3 and p-Akt1 were determined by western blot analysis (Fig. 10), with mock-infected cells serving as the sham control. In the Akt1-overexpressing cell line, CVB3 infection increased the LC3-II/LC3-I ratio $(\mathrm{p}<0.05)$. Moreover, the LC3-II/LC3-I ratio increased to greater levels when the infected cells were pre-treated with MK2206. In addition, under uninfected conditions, the LC3-II/LC3-I ratio was higher in the empty vector cells compared with that in the Akt1-overexpressing cells $(\mathrm{p}<0.05)$.

\section{Discussion}

Autophagy is a significant cellular catabolic process in which long-lived proteins and damaged organelles are degraded in lysosomes. Autophagy also serves as an important function in innate host defense by eliminating intracellular pathogens $(39,40)$. Autophagy begins with the generation of phagophores, which elongate and self-fuse to form double-membrane vesicles, known as autophagosomes (41). The formation of phagophores and autophagosomes is meditated by autophagy-related genes (ATGs). Microtubule-associated protein LC3, the mammalian homolog of yeast ATG8, is one well-studied marker of the presence of autophagic membranes (42). LC3-I localizes to the cytoplasm when autophagy occurs. LC3-I is conjugated with phosphatidylethanolamine (PE) to form lipidated LC3-PE, also known as LC3-II. The p62 protein interacts with LC3-II to target cargo to the autophagosomes for degradation. Autophagosomes fuse with endosomes to generate amphisomes, which obtain vacuolar-ATPase and become acidic. Subsequently, amphisomes fuse with incoming lysosomes to form autolysosomes. The cargo in the lumina of autolysosomes is then degraded by lysosomal proteases. The p62 protein can also be used to indicate the level of autophagic flux, as this protein is degraded along with LC3-II during complete autophagy $(43,44)$. However, during CVB3 infection, viral protease $2 \mathrm{~A}^{\text {pro }}$ can also account for p62 cleavage (45).

CVB3 infection induces autophagic reactions in vivo and in vitro, but the exact mechanism is unclear. The $\mathrm{PI} 3 \mathrm{~K} / \mathrm{Akt} / \mathrm{mTOR}$ pathway plays a vital role in the regulation of autophagy (46). Class I PI3K is the traditional upstream activator of mTOR. As the major upstream modulator, the PI3K pathway regulates autophagy by phosphorylating Akt. Activation of Akt activates mTORC1 by phosphorylation of tuberous sclerosis complex (TSC)2, causing the inhibition of the GTPase-activating protein (GAP) domain of TSC2 leading to the activation of mTORC1 and inhibition of autophagy (47).

Our research demonstrated that CVB3 induces autophagosome formation in HeLa cells, including an increase in the LC3-II/LC3-I ratio, LC3 punctate dot formation as demonstrated by immunofluorescence and autophagosome-like vesicle formation as detected via TEM. These data suggested a successful induction of autophagy caused by CVB3. Previous 
in vitro studies suggested that CVB3 could inhibit the degradation of virus-induced autophagosomes by blocking their fusion with lysosomes (48). In our time point experiment of CVB3 infection, autophagy was rapidly activated after viral infection and reached its peak at 5 h.p.i. accompanied by an obvious decrease of p62. Then, p62 increased as the time of infection increased. We did not detect an obvious expression of VP1 until 7 h.p.i. We then hypothesized that autophagy may play a protective role at the beginning of virus infection given that activated autophagy was accompanied by a degradation in p62. This may explain why we were unable to detect VP1 until 7 h.p.i. Then, the increase in autophagosomes affected the function of lysosomes, resulting in accumulation of autophagosomes, as demonstrated by the increase in p62. However, we did not observe a continuous increase in p62 as the infected time increased, which is inconsistent with previous findings (21). We believe that different cell types and different infection times accounted for this discrepancy. Moreover, during CVB3 infection, viral protease $2 \mathrm{~A}^{\text {pro }}$ is responsible for p62 cleavage (45). Thus, p62 expression in the present study may not accurately represent the level of p62 caused directly by CVB3 infection. Our findings simply imply a correlation between autophagy caused by CVB3 infection and protein degradation, and the details require further study.

The autophagic process may be subverted by viruses to provide a surface for RNA replication as the time of infection increases. At 24 h.p.i., modulation of the autophagic pathway (using rapamycin or ZSTK474) to enhance or reduce autophagy resulted in an increase and a decrease in CVB3 mRNA replication, respectively. It appears that the virus can subvert components of the autophagic machinery for their own benefit. To our surprise, the increased autophagy caused by rapamycin did not increase VP1 expression. We hypothesized that the inactivation of mTORC1 by rapamycin would inhibit cap-dependent mRNA translation. We observed a co-localization of LC3-positive puncta with LAMP-1 by confocal microscopy at 24 h.p.i., suggesting that CVB3 infection may induce autophagosomes to fuse with lysosomes/endosomes to form autolysosomes/amphisomes. Autophagosomes primarily fuse with lysosomes during CVB3 infection, excluding a small proportion of the autophagosomes that fuse with endosomes to form amphisomes (49). We inhibited lysosome function using chloroquine phosphate and monitored the levels of p62 and LC3 protein. After blockade of lysosome function, the LC3-II/LC3-I ratio and p62 protein levels increased significantly. These data revealed that at 24 h.p.i., autophagy may partly retain the function of promoting protein degradation (VP1) by lysosomes. Hence, the high expression of CVB3 mRNA did not contribute to the VP1 synthesis in cells treated with rapamycin.

In our research, we found that p-mTOR was altered during the first $3 \mathrm{~h}$ and did not keep pace with the change in LC3. The MEK/ERK pathway and the PI3K/Akt pathway cross-inhibit each other (50). Xin et al claimed that CVB3-induced autophagosome accumulation occurs via the ERK pathway (33). We hypothesized that autophagy caused by CVB3 infection was an outcome of multiple factors, including activation or inactivation of multiple signaling pathways. The balance or crosstalk among them may account for the difference. p-mTOR and p-Akt1 decreased at 24 h.p.i., and pre-treatment of virus-infected cells with ZSTK474 alleviated the LC3-II/LC3-I ratio caused by CVB3 infection. Inhibition of mTOR and Akt1 with rapamycin or MK2206 before virus infection promoted autophagy induced by CVB3 infection. We hypothesized that the incomplete blockade may explain this result. Additionally, rapamycin may have aggravated CVB3 mRNA replication in our study. Does the increased virus replication subsequently prompt autophagy? This question warrants further study. In addition, we found a significant phenomenon under uninfected conditions; the LC3-II/LC3-I ratio was increased in Akt1-overexpressing cells when compared with that in the empty vector cells. This finding suggests that the overexpression of Akt1 may inhibit basal autophagy in HeLa cells. Our previous research demonstrated that Akt1 overexpression promotes CVB3-induced apoptosis in HeLa cells, thus Akt1 overexpression may activate the crosstalk of autophagy and apoptosis, change the cell status or activate other signaling pathways during viral infection, thus explaining the differences between the two cell lines.

In summary, our study demonstrated that HeLa cells respond to CVB3 infection by increasing LC3 expression and autophagosome formation in vitro. We demonstrated that the inhibition of the PI3K/Akt/mTOR signaling pathway could affect the autophagic reaction induced by CVB3 infection, resulting in changes in viral infection. These findings may provide a better understanding of VMC pathogenesis.

\section{Acknowledgements}

We acknowledge the Institute of Oncology, Central South University that provided the HeLa cells, and the Center Laboratory at the Third Xiangya Hospital of the Central South University that provided the experimental equipment and technical guidance necessary to complete our study. This study was funded by the National Natural Science Foundation of China (grant no. 81570346).

\section{References}

1. Yajima T and Knowlton KU: Viral myocarditis: From the perspective of the virus. Circulation 119: 2615-2624, 2009.

2. Yajima T: Viral myocarditis: Potential defense mechanisms within the cardiomyocyte against virus infection. Future Microbiol 6: 551-566, 2011.

3. Bowles NE, Ni J, Kearney DL, Pauschinger M, Schultheiss HP, McCarthy R, Hare J, Bricker JT, Bowles KR and Towbin JA: Detection of viruses in myocardial tissues by polymerase chain reaction: Evidence of adenovirus as a common cause of myocarditis in children and adults. J Am Coll Cardiol 42: 466-472, 2003

4. Esfandiarei M and McManus BM: Molecular biology and pathogenesis of viral myocarditis. Annu Rev Pathol 3: 127-155, 2008.

5. Sin J, Mangale V, Thienphrapa W, Gottlieb RA and Feuer R: Recent progress in understanding coxsackievirus replication, dissemination, and pathogenesis. Virology 484: 288-304, 2015.

6. Liao YH, Xia N, Zhou SF, Tang TT, Yan XX, Lv BJ, Nie SF, Wang J, Iwakura Y, Xiao H, et al: Interleukin-17A contributes to myocardial ischemia/reperfusion injury by regulating cardiomyocyte apoptosis and neutrophil infiltration. J Am Coll Cardiol 59: 420-429, 2012.

7. Garmaroudi FS, Marchant D, Hendry R, Luo H, Yang D, Ye X, Shi $\mathrm{J}$ and McManus BM: Coxsackievirus B3 replication and pathogenesis. Future Microbiol 10: 629-653, 2015.

8. Chen Z, Yang L, Liu Y, Tang A, Li X, Zhang J and Yang Z: LY294002 and Rapamycin promote coxsackievirus-induced cytopathic effect and apoptosis via inhibition of PI3K/AKT/mTOR signaling pathway. Mol Cell Biochem 385: 169-177, 2014. 
9. He B: Viruses, endoplasmic reticulum stress, and interferon responses. Cell Death Differ 13: 393-403, 2006.

10. Zha X, Yue Y, Dong N and Xiong S: Endoplasmic reticulum stress aggravates viral myocarditis by raising inflammation through the IRE1-associated NF- $\mathrm{KB}$ pathway. Can J Cardiol 31: 1032-1040, 2015

11. Zhang HM, Ye X, Su Y, Yuan J, Liu Z, Stein DA and Yang D: Coxsackievirus B3 infection activates the unfolded protein response and induces apoptosis through downregulation of p58IPK and activation of CHOP and SREBP1. J Virol 84 8446-8459, 2010.

12. Liang $\mathrm{C}$ and Jung JU: Autophagy genes as tumor suppressors. Curr Opin Cell Biol 22: 226-233, 2010.

13. Sarkar S and Rubinsztein DC: Huntington's disease: Degradation of mutant huntingtin by autophagy. FEBS J 275: 4263-4270, 2008.

14. Cadwell K, Stappenbeck TS and Virgin HW: Role of autophagy and autophagy genes in inflammatory bowel disease. Curr Top Microbiol Immunol 335: 141-167, 2009.

15. Lerena MC, Vázquez CL and Colombo MI: Bacterial pathogens and the autophagic response. Cell Microbiol 12: 10-18,2010.

16. Tal MC and Iwasaki A: Autophagy and innate recognition systems. Curr Top Microbiol Immunol 335: 107-121, 2009.

17. Wong J, Zhang J, Si X, Gao G, Mao I, McManus BM and Luo H: Autophagosome supports coxsackievirus B3 replication in host cells. J Virol 82: 9143-9153, 2008.

18. Kubli DA and Gustafsson AB: Cardiomyocyte health: Adapting to metabolic changes through autophagy. Trends Endocrinol Metab 25: 156-164, 2014

19. Robinson SM, Tsueng G, Sin J, Mangale V, Rahawi S, McIntyre LL, Williams W, Kha N, Cruz C, Hancock BM, et al: Coxsackievirus B exits the host cell in shed microvesicles displaying autophagosomal markers. PLoS Pathog 10: e1004045, 2014.

20. Zhai X, Bai B, Yu B, Wang T, Wang H, Wang Y, Li H, Tong L, Wang Y, Zhang F, et al: Coxsackievirus B3 induces autophagic response in cardiac myocytes in vivo. Biochemistry (Mosc) 80: 1001-1009, 2015

21. Kemball CC, Alirezaei M, Flynn CT, Wood MR, Harkins S, Kiosses WB and Whitton JL: Coxsackievirus infection induces autophagy-like vesicles and megaphagosomes in pancreatic acinar cells in vivo. J Virol 84: 12110-12124, 2010.

22. Alirezaei M, Flynn CT, Wood MR and Whitton JL: Pancreatic acinar cell-specific autophagy disruption reduces coxsackievirus replication and pathogenesis in vivo. Cell Host Microbe 11: 298-305, 2012

23. Xin L, Xiao Z, Ma X, He F, Yao H and Liu Z: Coxsackievirus $B 3$ induces crosstalk between autophagy and apoptosis to benefit its release after replicating in autophagosomes through a mechanism involving caspase cleavage of autophagy-related proteins. Infect Genet Evol 26: 95-102, 2014.

24. Luo $\mathrm{H}$ and McManus BM: Is autophagy an avenue to modulate coxsackievirus replication and pathogenesis? Future Microbiol 7: 921-924, 2012

25. Mohankumar V, Dhanushkodi NR and Raju R: Sindbis virus replication, is insensitive to rapamycin and torin1, and suppresses $\mathrm{Akt} / \mathrm{mTOR}$ pathway late during infection in HEK cells. Biochem Biophys Res Commun 406: 262-267, 2011.

26. Ma J, Sun Q, Mi R and Zhang H: Avian influenza A virus H5N1 causes autophagy-mediated cell death through suppression of mTOR signaling. J Genet Genomics 38: 533-537, 2011.

27. Shelly S, Lukinova N, Bambina S, Berman A and Cherry S: Autophagy is an essential component of Drosophila immunity against vesicular stomatitis virus. Immunity 30: 588-598, 2009.

28. Huang H, Kang R, Wang J, Luo G, Yang W and Zhao Z: Hepatitis $\mathrm{C}$ virus inhibits AKT-tuberous sclerosis complex (TSC), the mechanistic target of rapamycin (MTOR) pathway, through endoplasmic reticulum stress to induce autophagy. Autophagy 9: 175-195, 2013.

29. Hu B, Zhang Y, Jia L, Wu H, Fan C, Sun Y, Ye C, Liao M and Zhou J: Binding of the pathogen receptor HSP90AA1 to avibirnavirus VP2 induces autophagy by inactivating the AKT-MTOR pathway. Autophagy 11: 503-515, 2015.
30. Kim J, Kundu M, Viollet B and Guan KL: AMPK and mTOR regulate autophagy through direct phosphorylation of Ulk1. Nat Cell Biol 13: 132-141, 2011

31. Vlahakis A and Powers T: A role for TOR complex 2 signaling in promoting autophagy. Autophagy 10: 2085-2086, 2014.

32. Bernard M, Dieudé M, Yang B, Hamelin K, Underwood K and Hébert MJ: Autophagy fosters myofibroblast differentiation through MTORC2 activation and downstream upregulation of CTGF. Autophagy 10: 2193-2207, 2014

33. Xin L, Ma X, Xiao Z, Yao H and Liu Z: Coxsackievirus B3 induces autophagy in HeLa cells via the AMPK/MEK/ERK and Ras/Raf/MEK/ERK signaling pathways. Infect Genet Evol 36: 46-54, 2015.

34. Levine B and Kroemer G: Autophagy in the pathogenesis of disease. Cell 132: 27-42, 2008.

35. Li X, Li Z, Zhou W, Xing X, Huang L, Tian L, Chen J, Chen C, Ma X and Yang Z: Overexpression of 4EBP1, p70S6K, Akt1 or Akt2 differentially promotes Coxsackievirus B3-induced apoptosis in HeLa cells. Cell Death Dis 4: e803-e809, 2013.

36. Li X, Zhang J, Chen Z, Yang L, Xing X, Ma X and Yang Z: Both PI3K- and mTOR-signaling pathways take part in CVB3-induced apoptosis of Hela cells. DNA Cell Biol 32: 359-370, 2013.

37. Klionsky DJ, Abeliovich H, Agostinis P, Agrawal DK, Aliev G, Askew DS, Baba M, Baehrecke EH, Bahr BA, Ballabio A, et al: Guidelines for the use and interpretation of assays for monitoring autophagy in higher eukaryotes. Autophagy 4: 151-175, 2008.

38. Tanida I, Minematsu-Ikeguchi N, Ueno T and Kominami E: Lysosomal turnover, but not a cellular level, of endogenous LC3 is a marker for autophagy. Autophagy 1: 84-91, 2005.

39. Gutierrez MG, Master SS, Singh SB, Taylor GA, Colombo MI and Deretic V: Autophagy is a defense mechanism inhibiting BCG and Mycobacterium tuberculosis survival in infected macrophages. Cell 119: 753-766, 2004.

40. Mizushima N, Levine B, Cuervo AM and Klionsky DJ: Autophagy fights disease through cellular self-digestion. Nature 451: 1069-1075, 2008.

41. Mizushima N: Autophagy: Process and function. Genes Dev 21: 2861-2873, 2007.

42. Eskelinen EL: Maturation of autophagic vacuoles in Mammalian cells. Autophagy 1: 1-10, 2005.

43. Klionsky DJ, Abdalla FC, Abeliovich H, Abraham RT, Acevedo-Arozena A, Adeli K, Agholme L, Agnello M, Agostinis P, Aguirre-Ghiso JA, et al: Guidelines for the use and interpretation of assays for monitoring autophagy. Autophagy 8 : 445-544, 2012

44. Lai JK, Sam IC and Chan YF: The autophagic machinery in enterovirus infection. Viruses 8: E32, 2016.

45. Shi J, Wong J, Piesik P, Fung G, Zhang J, Jagdeo J, Li X, Jan E and Luo $\mathrm{H}$ : Cleavage of sequestosome $1 / \mathrm{p} 62$ by an enteroviral protease results in disrupted selective autophagy and impaired NFKB signaling. Autophagy 9: 1591-1603, 2013.

46. Heras-Sandoval D, Pérez-Rojas JM, Hernández-Damián J and Pedraza-Chaverri J: The role of PI3K/AKT/mTOR pathway in the modulation of autophagy and the clearance of protein aggregates in neurodegeneration. Cell Signal 26: 2694-2701, 2014.

47. Yao H, Han X and Han X: The cardioprotection of the insulin-mediated PI3K/Akt/mTOR signaling pathway. Am J Cardiovasc Drugs 14: 433-442, 2014.

48. Taylor MP and Jackson WT: Viruses and arrested autophagosome development. Autophagy 5: 870-871, 2009.

49. Shi X, Chen Z, Tang S, Wu F, Xiong S and Dong C: Coxsackievirus B3 infection induces autophagic flux, and autophagosomes are critical for efficient viral replication. Arch Virol 161: 2197-2205, 2016.

50. Hoeflich KP, O'Brien C, Boyd Z, Cavet G, Guerrero S, Jung K, Januario T, Savage H, Punnoose E, Truong T, et al: In vivo antitumor activity of MEK and phosphatidylinositol 3-kinase inhibitors in basal-like breast cancer models. Clin Cancer Res 15 : 4649-4664, 2009. 